\title{
High leukocyte mtDNA content contributes to poor prognosis through ROS-mediated immunosuppression in hepatocellular carcinoma patients
}

\author{
Xianli He ${ }^{1, *}$, Falin Qu ${ }^{1,2, *}$, Feng Zhou ${ }^{1}$, Xingchun Zhou ${ }^{2}$, Yibing Chen ${ }^{2}$, Xu Guo ${ }^{2}$, Jibin \\ $\mathbf{L i}^{2}$, Qichao Huang ${ }^{2}$, Yefa Yang ${ }^{3}$, Zhuomin Lyu ${ }^{4}$, Hongxin Zhang ${ }^{4}$, Jinliang Xing ${ }^{2}$ \\ ${ }^{1}$ Department of General Surgery, Tangdu Hospital, The Fourth Military Medical University, Xi'an 710032, China \\ ${ }^{2}$ State Key Laboratory of Cancer Biology and Experimental Teaching Center of Basic Medicine, The Fourth Military Medical \\ University, Xi'an 710032, China \\ ${ }^{3}$ Department of Radioactive Intervention, Eastern Hepatobiliary Surgery Hospital, The Second Military Medical University, \\ Shanghai 200438, China \\ ${ }^{4}$ Department of Pain Treatment, Tangdu Hospital, The Fourth Military Medical University, Xi'an 710032, China \\ *These authors have contributed equally to this work \\ Correspondence to: Jinliang Xing, e-mail: xingjl@fmmu.edu.cn \\ Keywords: mitochondrial DNA content, hepatocellular carcinoma, prognosis, reactive oxygen species, immunosuppression \\ Received: November 10,2015 Accepted: February 23, $2016 \quad$ Published: March 14, 2016
}

\section{ABSTRACT}

Compelling epidemiological evidences indicate a significant association between leukocyte mitochondrial DNA (mtDNA) content and incidence risk of several malignancies, including hepatocellular carcinoma (HCC). However, whether leukocyte mtDNA content affect prognosis of HCC patients and underlying mechanism has never been explored. In our study, leukocyte mtDNA content was measured in 618 HCC patients and its prognostic value was analyzed. Moreover, we detected the immunophenotypes of peripheral blood mononuclear cells (PBMCs) and plasma concentrations of several cytokines in $40 \mathrm{HCC}$ patients and assessed the modulating effects of mtDNA content on immunosuppression in cell models. Our results showed that HCC patients with high leukocyte mtDNA content exhibited a significantly worse recurrence-free survival (RFS) and overall survival (OS) than those with low leukocyte mtDNA content. Leukocyte mtDNA content and TNM stage exhibited a notable joint effect in prognosis prediction. Furthermore, we found that patients with high leukocyte mtDNA content exhibited a higher frequency of CD4 ${ }^{+}$CD25 ${ }^{+}{ }^{+} O X P 3^{+}$ regulatory $T$ (Treg) cells and lower frequency of NK cells in PBMCs and had higher TGF- $\beta 1$ and lower TNF- and IFN- plasma concentration when compared with those with low leukocyte mtDNA content, which suggests an immunosuppressive status. High leukocyte mtDNA content significantly enhanced the ROS-mediated secretion of TGF- $\beta 1$, which accounted for higher Treg and lower NK frequency in PBMCs. In a conclusion, our study for the first time demonstrates that leukocyte mtDNA content is an independent prognostic marker complementing TNM stage and associated with an ROS-mediated immunosuppressive phenotype in HCC patients.

\section{INTRODUCTION}

Hepatocellular carcinoma (HCC) is the sixth most common malignant disease and the third leading cause of cancer-related death worldwide [1]. Despite recent improvements in diagnosis and treatment, clinical outcome of HCC is still disappointing [2]. Due to the molecular and genetic heterogeneity, HCC patients with the similar clinical and pathological features often exhibit distinct outcomes [3]. Therefore, it is urgent to identify new molecular biomarkers to complement TNM staging system for more precise prognostic prediction of HCC, and thus benefit for individualized therapy. 
Mitochondria plays multiple roles in energy metabolism and cellular homeostasis, mainly including the generation of ATP and reactive oxygen species (ROS) [4]. Human mitochondrial DNA (mtDNA) is a circular doublestranded DNA molecule of 16,569 base pairs [5]. The mtDNA content (also referred as mtDNA copy number) varies from several hundred to more than 10,000 copies per cell, depending on the cell type [6]. The mtDNA content may undergo significant changes under diverse internal or external microenvironments, which can lead to impairment of the OXPHOS system and the enhanced generation of ROS. This scenario has been proposed to contribute to the initiation and progression of tumors [7].

Quantitative changes in mtDNA content have been observed in many types of malignancies, such as HCC, gastric cancer, head and neck cancer and colorectal cancer (CRC) [8-11]. Previous studies have also revealed that altered mtDNA content in tumor tissues was associated with tumor stage, prognosis, and treatment response, again in a cancer type-specific manner [10, 12-14]. Yamada et al. have found that HCC tissues have a reduced copy number of mtDNA when compared with paired non-tumor tissues, which is associated with malignant potential of HCC [15]. In recent years, there are a series of reports on the association of the mtDNA content in peripheral blood lymphocytes (PBLs) with cancer susceptibility [16-20]. However, only two studies have indicated that mtDNA content in PBLs is associated with breast cancer progression and CRC prognosis [21, 22]. The effect of mtDNA content in PBLs on HCC patient prognosis and its underlying mechanism has not been explored.

Herein, we measured mtDNA content in PBLs from HCC patients and assessed its prognostic value. Furthermore, we explored the potential immune-related mechanism underlying the prognostic effects of leukocyte mtDNA content. To the best of our knowledge, this is the first study to investigate the prognostic significance of leukocyte mtDNA content in HCC patients, which may benefit for future improvement of treatment.

\section{RESULTS}

\section{Characteristics of patient populations and distribution of mtDNA content}

Demographic and clinical characteristics of HCC patients were summarized in Supplementary Table 1. At latest follow-up, 373 patients developed recurrence and 240 died. The median value (range) of normalized mtDNA content was [0.98 (0.12-3.70)] in total samples and no significant difference was observed for mtDNA content among training [0.99 (0.16-3.39)] and validation [0.98 (0.12-3.70)] cohorts $(P=0.987)$ (Supplementary Table 1). We then compared mtDNA content between various epidemiological and clinical characteristics in training cohort, validation cohort and total patients (Supplementary Table 2). No significant difference of mtDNA content was found between patients with different age, sex, HBsAg status, tumor size, number of tumors, PVT, TNM stage, differentiation, and AFP level ( $P$ values ranging from 0.092 to 0.996 ). However, mtDNA content was significantly higher in patients with recurrence than those in patients without recurrence in total cohort $(P<$ $0.001)$. Similar result was observed in patients who died when compared with those who were alive $(P=0.001)$.

\section{Prognostic analysis for leukocyte mtDNA content in HCC patients}

To find the optimal cutoff point of mtDNA content value that best distinguish the OS and RFS for HCC patients, we constructed the ROC curves for events based on mtDNA content values in the training cohort and found a mtDNA content value of 0.98 to be the best cutoff points for OS and RFS $(P=0.004$ and 0.002 , Supplementary Figure 1). This value was used as a uniform cutoff point to dichotomize patients into two subgroups with high or low mtDNA content in all the subsequent analyses. Then, we analyzed the prognostic effect of mtDNA content on OS and RFS. Univariate (Supplementary Table 3) and multivariate analyses (Table 1) indicated that high leukocyte mtDNA content was significantly associated with poor OS and RFS of patients in training cohort $(\mathrm{HR}=$ $1.89,95 \% \mathrm{CI}=1.05-3.39, P=0.034 ; \mathrm{HR}=1.90,95 \% \mathrm{CI}=$ $1.12-3.22, P=0.018$, respectively) and validation cohort $(\mathrm{HR}=1.88,95 \% \mathrm{CI}=1.38-2.58, P<0.001 ; \mathrm{HR}=1.84$, $95 \% \mathrm{CI}=1.22-2.95, P<0.001$, respectively). Combining two patient cohorts, high leukocyte mtDNA content was associated with a 1.89 -fold increased risk of death $(95 \%$ CI, 1.44 - 2.48) and 1.86-fold increased risk of recurrence (95\% CI, 1.26 - 2.98), respectively (Table 1). KaplanMeier survival function analysis showed that patients with high mtDNA content had a shorter OS and RFS than did those with low mtDNA content in the training (log-rank $P=0.007$ and 0.003 , respectively, Figure 1A), validation (both log-rank $P<0.001$, Figure 1B) and total cohort (both log-rank $P<0.001$, Figure 1C).

\section{Prognostic prediction of leukocyte mtDNA content complementing to TNM stage}

Considering prognosis heterogeneity in same TNM stage, we thus evaluated whether leukocyte mtDNA content can improve prognostic prediction based on TNM stage in the total HCC patient populations. As shown in Figure 2A and 2B, ROC analysis showed that the combined model of TNM stage and mtDNA had the largest AUC, indicating a significantly better prediction efficacy of OS and RFS than either TNM stage or mtDNA only model. We then compared the OS and RFS of HCC 
Table 1: Multivariable Cox regression analysis of prognosis for HCC patients

\begin{tabular}{|c|c|c|c|c|c|c|}
\hline \multirow{2}{*}{ Variables } & \multicolumn{2}{|c|}{ Training cohort } & \multicolumn{2}{|c|}{ Validation cohort } & \multicolumn{2}{|c|}{ Total cohort } \\
\hline & HR $(95 \%$ CI $)$ & Pvalue & HR (95\% CI) & Pvalue & HR $(95 \%$ CI $)$ & Pvalue \\
\hline \multicolumn{7}{|l|}{ Overall survival } \\
\hline Age $(>52$ vs. $\leq 52)$ & $1.34(0.77-2.33)$ & 0.305 & $1.13(0.83-1.54)$ & 0.443 & $1.13(0.86-1.48)$ & 0.368 \\
\hline Sex (male vs. female) & $1.19(0.36-3.99)$ & 0.774 & $1.14(0.71-1.85)$ & 0.582 & $1.29(0.83-1.99)$ & 0.253 \\
\hline HBsAg (postive vs. negative) & $1.69(0.83-3.47)$ & 0.149 & $1.14(0.65-1.99)$ & 0.657 & $1.19(0.84-1.68)$ & 0.339 \\
\hline TNM stage ( III+IV vs. I+II) & $3.01(1.63-5.58)$ & $<0.001$ & $2.29(1.55-3.37)$ & $<0.001$ & $1.74(1.21-2.51)$ & 0.003 \\
\hline $\begin{array}{l}\text { Differentiation (Poor vs. } \\
\text { Moderate+Well) }\end{array}$ & $2.55(1.47-4.93)$ & $<0.001$ & $2.11(1.25-3.69)$ & $<0.001$ & $1.98(1.17-3.08)$ & 0.008 \\
\hline $\operatorname{Serum} \operatorname{AFP}(\geq 200 \mu \mathrm{g} / \mathrm{L} v s .<200 \mu \mathrm{g} / \mathrm{L})$ & $2.42(1.31-4.45)$ & 0.005 & $1.81(1.31-2.49)$ & $<0.001$ & $1.73(1.31-2.28)$ & $<0.001$ \\
\hline mtDNA content (high vs. low) & $1.89(1.05-3.39)$ & 0.034 & $1.88(1.38-2.58)$ & $<0.001$ & $1.89(1.44-2.48)$ & $<0.001$ \\
\hline \multicolumn{7}{|l|}{ Recurrence-free survial } \\
\hline Age $(>52$ vs. $\leq 52)$ & $1.08(0.66-1.78)$ & 0.75 & $0.98(0.77-1.25)$ & 0.874 & $1.00(0.81-1.24)$ & 0.99 \\
\hline Sex (male vs. female) & $1.12(0.39-3.16)$ & 0.836 & $1.26(0.87-1.83)$ & 0.225 & $1.31(0.93-1.85)$ & 0.125 \\
\hline HBsAg (postive $v s$. negative) & $1.55(0.82-2.96)$ & 0.181 & $1.02(0.67-1.54)$ & 0.93 & $1.17(0.75-1.81)$ & 0.496 \\
\hline TNM stage ( III+IV vs. I+II) & $4.12(2.36-7.20)$ & $<0.001$ & $1.83(1.34-2.51)$ & $<0.001$ & $1.72(1.28-2.32)$ & $<0.001$ \\
\hline $\begin{array}{l}\text { Differentiation (Poor vs. } \\
\text { Moderate+Well) }\end{array}$ & $2.29(1.38-3.85)$ & $<0.001$ & $2.06(1.25-3.84)$ & $<0.001$ & $1.97(1.17-2.93)$ & 0.009 \\
\hline Serum AFP $(\geq 200 \mu \mathrm{g} / \mathrm{L} v s .<200 \mu \mathrm{g} / \mathrm{L})$ & $1.84(1.11-3.04)$ & 0.018 & $1.61(1.25-2.07)$ & $<0.001$ & $1.61(1.29-2.02)$ & $<0.001$ \\
\hline mtDNA content (high vs. low) & $1.90(1.12-3.22)$ & 0.018 & $1.84(1.22-2.95)$ & $<0.001$ & $1.86(1.29-2.98)$ & $<0.001$ \\
\hline
\end{tabular}

Notes: mtDNA, mitochondrial DNA; HR: hazard ratio; 95\% CI, 95\% confidence interval. Significant $P$ value was in bold. We calculated hazaed ratios and $P$ values with an adjusted multivariate Cox proportional hazards regression model, including age, sex, HBsAg, TNM stage, differentiation, serum AFP and mtDNA content as covariates.

patients in different subgroups divided by TNM stage together with mtDNA content by Kaplan-Meier survival analysis and found that patients with high mtDNA content at TNM stage III/IV exhibited the worst OS and RFS, whereas those with low mtDNA content at TNM stage I/II had the best OS and RFS (both log-rank $P<0.001$ ) (Figure $2 \mathrm{C}$ and $2 \mathrm{D})$. In the multivariate Cox regression analysis, we found that patients with high mtDNA content at TNM stage III/IV had the highest risk of death and recurrence when using patients with low mtDNA content and at lower TNM stage I/II as reference $(\mathrm{HR}=6.12,95 \% \mathrm{CI}=4.17$ 8.98 for OS; $\mathrm{HR}=4.29,95 \% \mathrm{CI}=3.12-5.88$ for $\mathrm{RFS}$ ) (Supplementary Table 4).

\section{Immunophenotypes of PBMCs and plasma concentration of cytokines in $\mathrm{HCC}$ patients with different leukocyte mtDNA content}

To explore the potential mechanisms underlying leukocyte mtDNA content as a independent prognostic predictor for HCC patients, we first examined the associations between subtypes of lymphocytes in
PBMCs and leukocyte mtDNA content in $40 \mathrm{HCC}$ patients, whose demographic and clinical characteristics were summarized in supplementary Table 5. FACS analyses showed that patients with high leukocyte mtDNA content which was defined by using 0.98 as cutoff point had significantly higher percentage of $\mathrm{CD}^{+}$ T cells $(41.6 \%$ vs. $35.3 \%, P=0.042$, Figure $3 \mathrm{~A})$ and $\mathrm{CD}^{+} \mathrm{CD} 25^{+} \mathrm{FOXP} 3^{+}$regulatory $\mathrm{T}$ (Treg) cells $(5.3 \%$ vs. $9.3 \%, P=0.004$, Figure 3B) and lower percentage of natural killer (NK) cells $(22.4 \%$ vs. $16.6 \%, P=0.028$, Figure 3C) than those with low leukocyte mtDNA content. In addition, patients with high mtDNA content had significantly higher plasma TGF- $\beta 1$ concentration (97.3 pg/ml vs. $162.1 \mathrm{pg} / \mathrm{ml}, P=0.013$, Figure 3D) and lower TNF- $\alpha$ and IFN- $\gamma$ concentration $(358.7 \mathrm{pg} / \mathrm{ml} v s$. $258.0 \mathrm{pg} / \mathrm{ml}, P=0.024$ and $32.9 \mathrm{pg} / \mathrm{ml}$ vs. $15.7 \mathrm{pg} / \mathrm{ml}, P$ $=0.002$, respectively, Figure $3 \mathrm{E}$ and $3 \mathrm{~F}$ ) than those with low mtDNA content. However, there was no significant difference in the frequency of other immune cells or the concentration of other cytokines between patients with high and low mtDNA content (Supplementary Figure 2). 

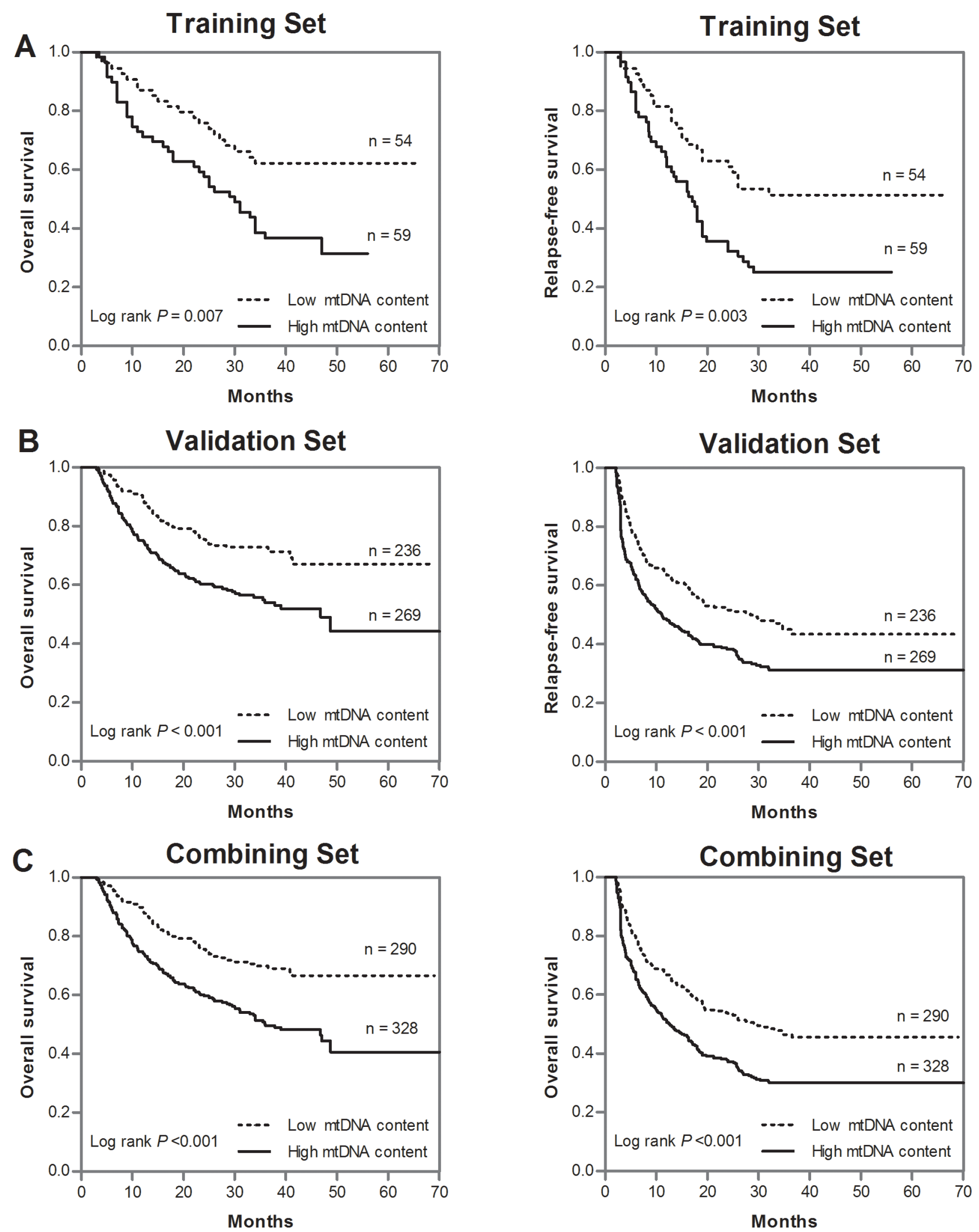

Figure 1: Kaplan-Meier estimates of OS and RFS in HCC patients by leukocyte mtDNA content. A. OS and RFS in the training cohort; B. OS and RFS in the validation cohort; C. OS and RFS in the total cohort. 


\section{Elevated leukocyte mtDNA content induced an immunosuppressive phenotype by ROS- mediated secretion of TGF- $\beta 1$}

To further identify the cause-effect relationship between mtDNA content variation and immunophenotypes of PBMCs or plasma concentration of cytokines, mitochondrial transcription factor TFAM was overexpressed to induce the elevation of mtDNA content in both Jurkat and H9 cells (Figure 4A). Then, intracellular ROS was found to be significantly increased in both cells (Figure 4B and 4C). We then measured the secretion of cytokines and found that TFAM overexpression (i.e. higher mtDNA content) significantly increased the concentration of TGF- $\beta 1$ in culture supernatants of H9 and Jurkat, whereas the reducing reagent $N$-acetyl cysteine (NAC), a ROS scavenger, reversed this effect (Figure $4 \mathrm{D}$ and $4 \mathrm{E}$ ). Moreover, $\mathrm{H}_{2} \mathrm{O}_{2}$ directly induced TGF- $\beta 1$

\section{$\mathbf{A}$}

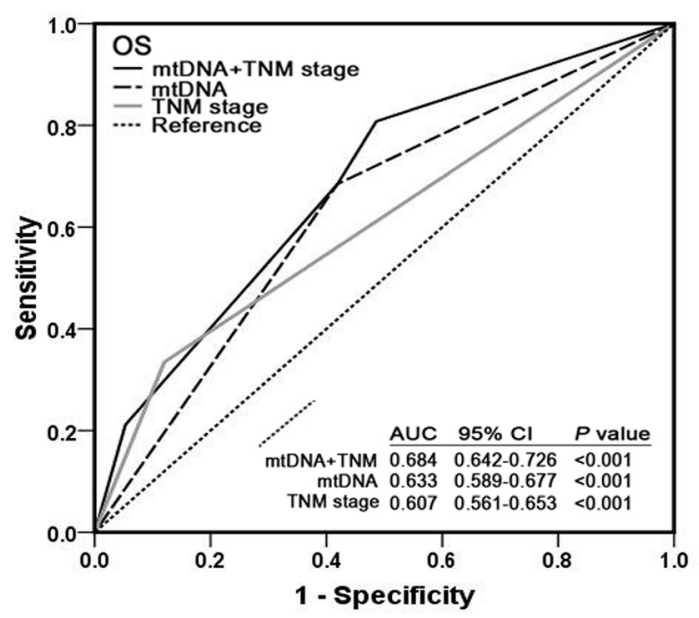

C

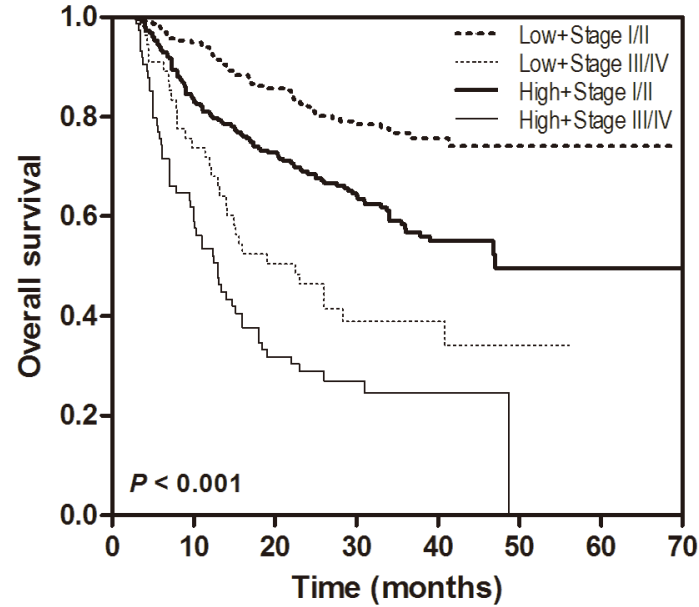

secretion and suppressed TNF- $\alpha$ and IFN- $\gamma$ secretion in PBMCs from healthy donors, while NAC significantly counteracted the effects of $\mathrm{H}_{2} \mathrm{O}_{2}$ (Figure 5A). TGF- $\beta 1$ has been reported to suppresses the secretion of TNF- $\alpha$, an important inhibitor of Treg activation [26], as well as the secretion of IFN- $\gamma$. Thus, we assessed the impact of culture supernatants from Jurkat or H9 cells with different mtDNA content on the PBMCs from healthy donors and found that supernatants from both TFAM-overexpressed Jurkat and $\mathrm{H} 9$ cells significantly inhibited TNF- $\alpha$ and IFN- $\gamma$ secretion (Figure 5B). NK cell frequency was significantly decreased in PBMCs by $\mathrm{H}_{2} \mathrm{O}_{2}$ treatment or TGF- $\beta 1$ treatment and culturing with supernatants from TFAM-overexpressed cells (Figure 5C). Furthermore, Treg frequency was significantly elevated in PBMCs by treatment with $\mathrm{H}_{2} \mathrm{O}_{2}$ or TGF- $\beta 1$ and culturing with supernatants from TFAM-overexpressed cells (Figure 5D). All our findings provide the evidence that higher mtDNA
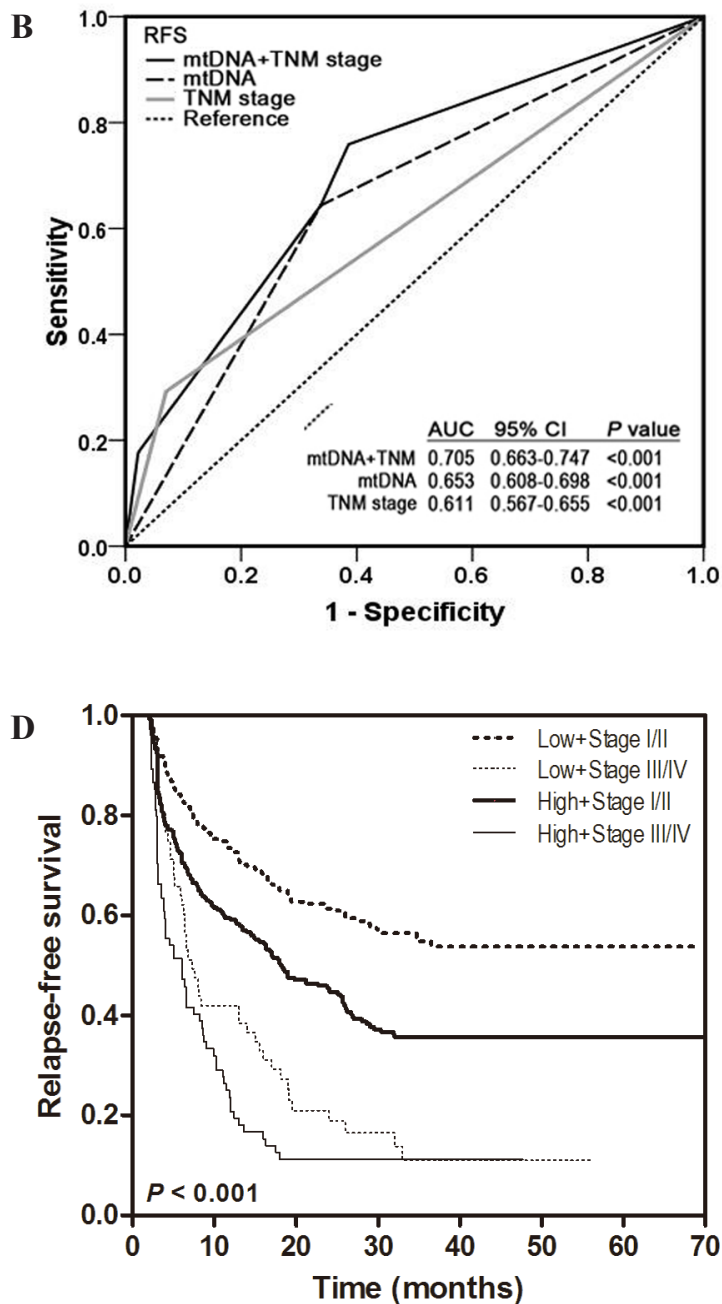

Figure 2: Joint prognostic values of leukocyte mtDNA content and TNM stage in HCC patients. A-B. ROC curve analysis was used to compare the sensitivity and specificity for prediction of OS and RFS by the combined mtDNA content and TNM stage model, mtDNA content alone model and TNM stage alone model. C-D. Kaplan-Meier curves of OS and RFS in subgroups stratified by mtDNA content and TNM stage. Hazards ratios and 95\% CIs were calculated by multivariate Cox proportional hazards regression model, adjusted for age, sex, HBsAg, differentiation, TNM stage, serum AFP and mtDNA content as covariates. 
content induces the quantitative variation of Treg and NK cells via ROS-mediated secretion of TGF- $\beta 1$, thereby contributing to the worse prognosis of HCC (Figure 5E).

\section{DISCUSSION}

A growing number of epidemiological studies have demonstrated that leukocyte mtDNA content is closely related to risk of various cancers. For instance, several previous studies have reported that leukocyte mtDNA content is associated with the risks of breast, pancreas, liver, esophageal, colon and lung cancers [19, 20, 27, 28]. Moreover, Xia et al. have demonstrated that leukocyte mtDNA content is associated with the T stage of breast cancer, indicating that high mtDNA content may facilitate the progression of cancer [21]. In this study, we for the first time identified leukocyte mtDNA content as an independent prognostic factor for $\mathrm{HCC}$, indicating that high leukocyte mtDNA content was associated with poor OS and RFS of patients. In comparison, Hashad et al. have reported that HCV-related HCC patients with multicentric hepatic lesions have significantly lower mtDNA content than those with less advanced disease, suggesting that functional roles of mtDNA copy number may be diseasespecific [16]. Collectively, these findings support the point of view that mtDNA content variations are involved in the progression of cancer, although the molecular mechanisms underlying the regulation of mtDNA copy number, especially in lymphocytes from cancer patients, remains to be largely unclear.

Current TNM staging system plays an important role in the prognosis prediction and treatment decisionmaking of HCC patients. However, patients in the same stage undergoing same therapeutic regimen show heterogeneous outcome. Therefore, introduction of novel biomarkers into the TNM staging system may improve the prognosis prediction of HCC patients. As shown in our study, integration of leukocyte mtDNA into TNM stage-based prognosis prediction models significantly improved the prediction power of overall survival of HCC patients. Once HCC patients with poor prognosis can be effectively predicted, potential therapeutic interventions will be applied, such as frequent imaging scan (CT or MRI) and AFP level monitoring, treatment with adjuvant transarterial chemoembolization (TACE) or Sorafenib (an orally active multikinase inhibitor) [29].

The biological mechanism behind the association between leukocyte mtDNA content alteration and HCC progression remains to be clarified. Previous studies have demonstrated that either increase in Tregs or decrease in NK cell frequency is significantly associated with poor survival of HCC patients [30]. It is generally considered
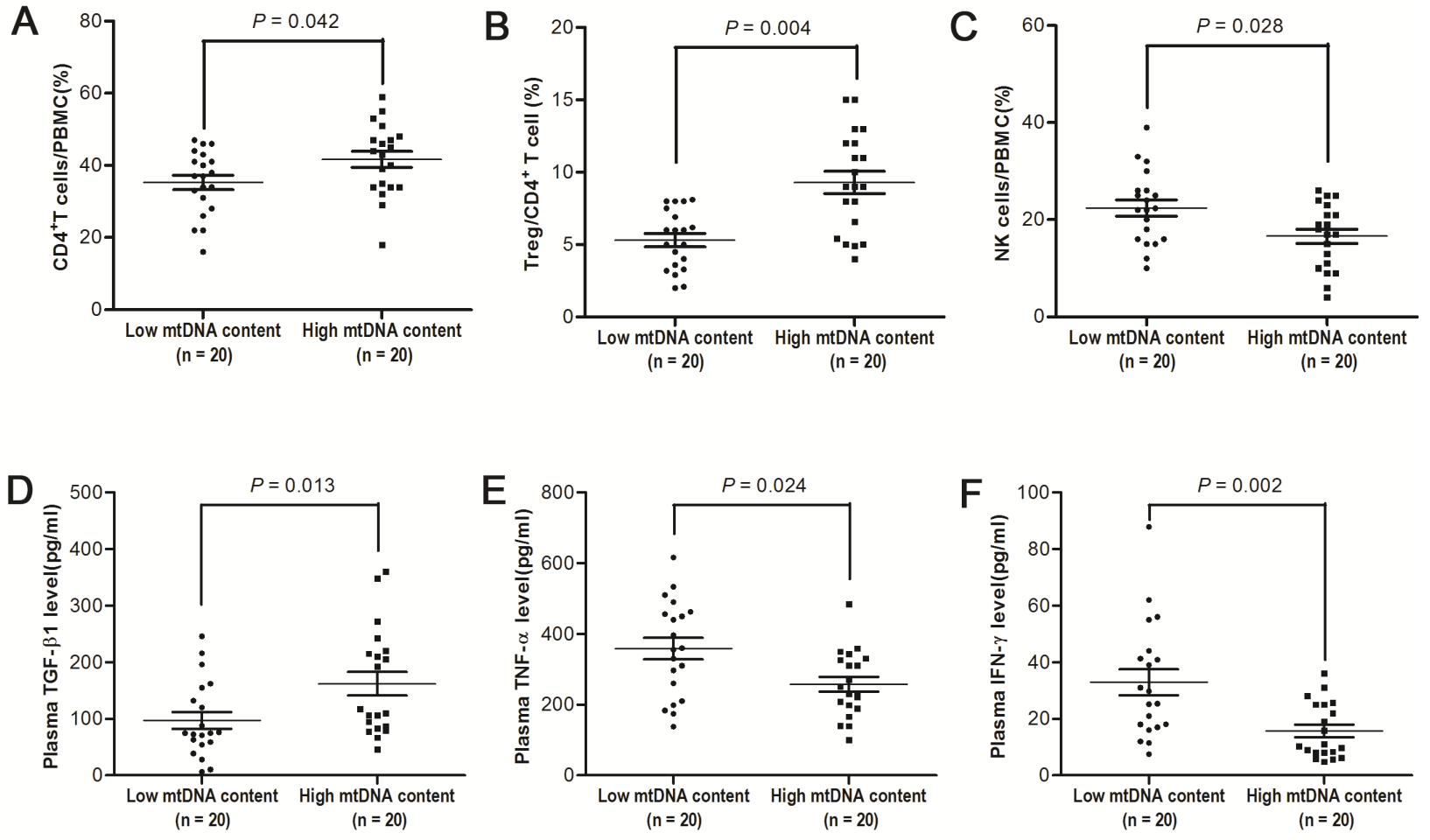

Figure 3: Immunophenotypes of PBMCs and plasma concentration of cytokines in HCC patients with different leukocyte mtDNA content. A-C. Flow cytometry analyses for percentage of CD4 ${ }^{+}$in PBMCs, Treg cells in CD4 $4^{+}$T cells and NK-cells in PBMCs from HCC patients with high and low mtDNA content (both $\mathrm{n}=20$ ). D-F. ELISA analyses for the plasma concentrations of TGF- $\beta 1$, TNF- $\alpha$ and IFN- $\gamma$ from HCC patients with high and low mtDNA content (both $\mathrm{n}=20$ ). 
that NK cells exert immunosurveillance against cancer cells, while Tregs inhibit anticancer immunity. In present study, we found that HCC patients with high leukocyte mtDNA content had an increased percentage of Tregs and the decreased percentage of NK-cells in peripheral blood, suggesting that mtDNA content may be involved in the immunosuppression of HCC patients. Moreover, it has been reported that IFN- $\gamma$ and TNF- $\alpha$ levels in plasma are associated good prognosis of HCC $[31,32]$, while TGF- $\beta 1$ plasma concentration is associated with poor prognosis of HCC [33]. IFN- $\gamma$ is one of the key mediator of cytotoxicity secreted by NK cells, while TNF- $\alpha$ enhances the functions of NK cells and suppress the functions of Tregs. As an important immunosuppressive cytokine, TGF- $\beta 1$ has a wide spectrum of inhibitory functions, including promoting Treg proliferation and differentiation, as well as inhibiting the expansion and functions of helper $\mathrm{T}$ cells and killer cells. In this study, our data also showed that HCC patients with high leukocyte mtDNA content had lower plasma TNF- $\alpha$ and IFN- $\gamma$ but higher TGF- $\beta 1$ concentrations, indicating that mtDNA content may affect cytokine secretion of immune cells.

In mammalian cells, mitochondria are the major source of reactive oxygen species (ROS) [34]. Generally, the increase in mtDNA content is related to an increase of ROS production [35]. Previous studies
A
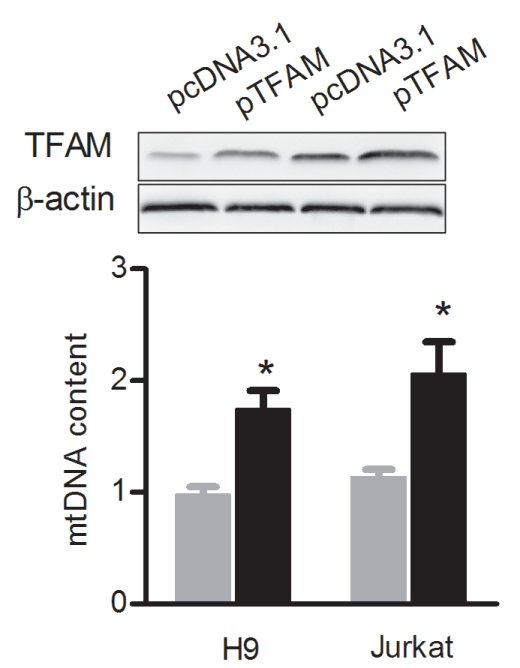

D
B

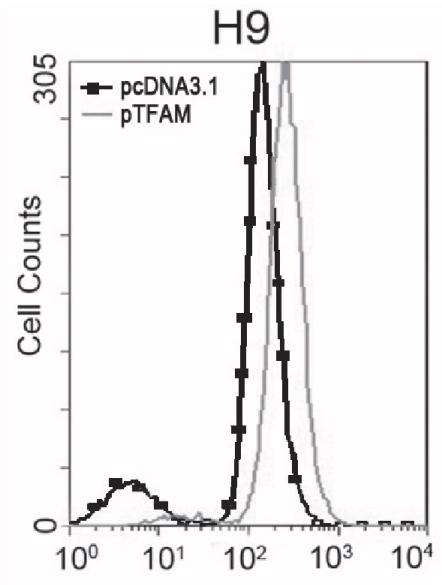

C

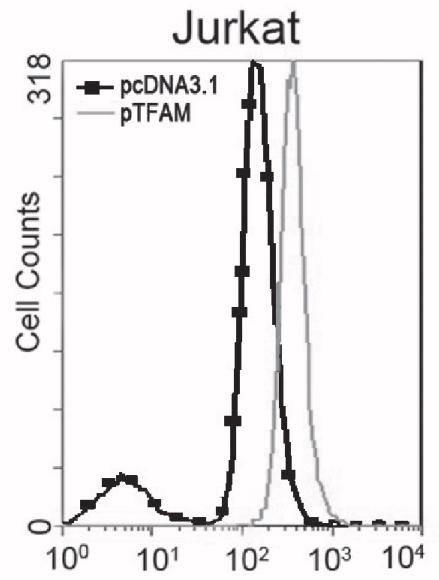

$\mathbf{E}$
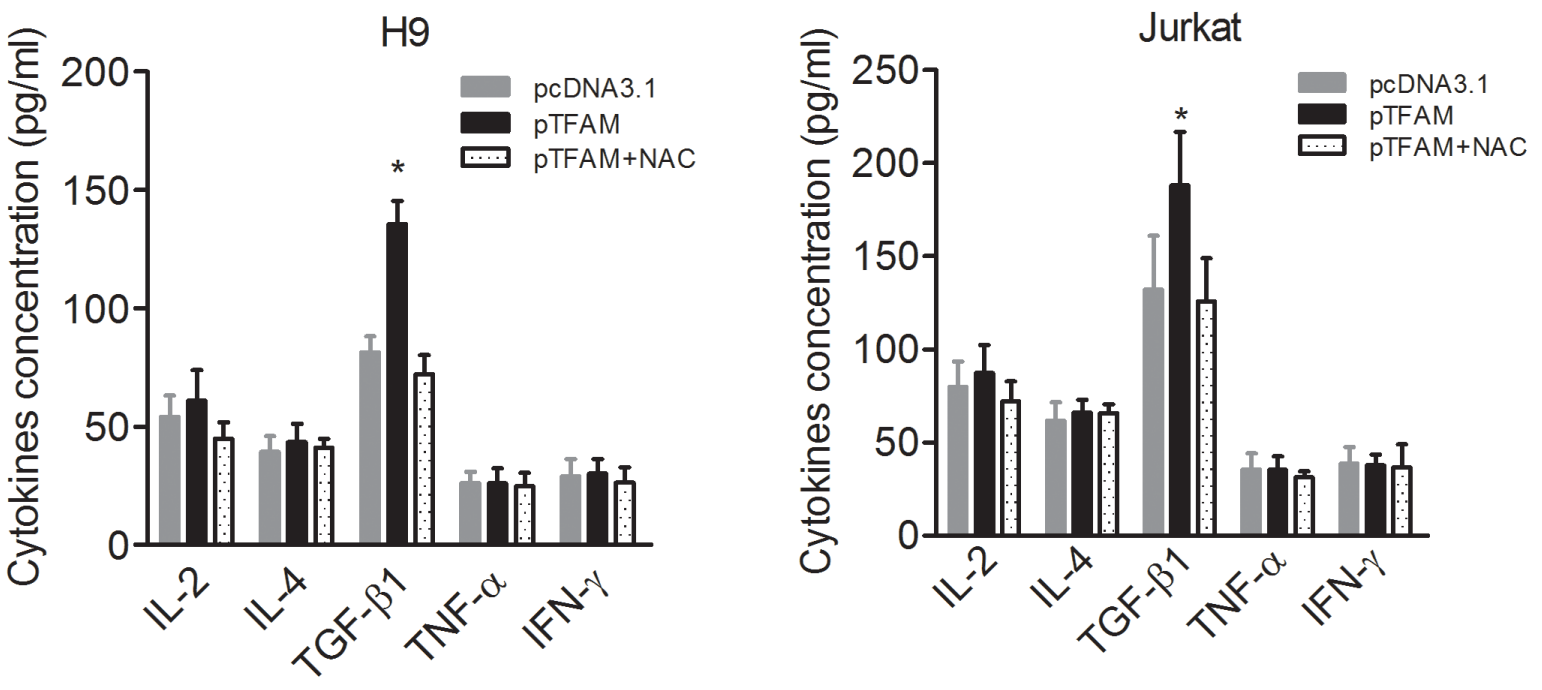

Figure 4: Elevated mtDNA content induced the production of ROS to increase the secretion of TGF- $\beta 1$ in T cells. A. TFAM expression at protein level detected by Western blot, and relative mtDNA content measured by real-time PCR-based method in H9 and Jurkat cells 48 hours after transfection with pTFAM or pcDNA3.1. B. and C. ROS measured by staining with $\mathrm{H}_{2}$ DCF-DA and then analyzed by flow cytometer in H9 and Jurkat cells with different plasmid transfection or treatment. D. Cytokines in culture supernatants measured by ELISA in H9 cells with different plasmid transfection or treatment. E. Cytokines in culture supernatants measured by ELISA in Jurkat cells with different plasmid transfection or treatment. ${ }^{*} P<0.05$, compared with related controls. 
have reported that mitochondrial ROS is an important inducer of TGF- $\beta 1$ [36], a key cytokine accounting for the differentiation, proliferation and functions of Treg and NK cells. Consistently, our in vitro cell model analysis indicated that high mtDNA content induced ROS generation to increase the secretion of TGF- $\beta 1$ in T cell lines. Mitochondria-derived ROS play an important role in the immune functions of $\mathrm{T}$ cells, such as activation of naive $T$ cells, cytokine secretion of effect $T$ cells, and apoptosis of activated $\mathrm{T}$ cells [37]. Kraaij et al. have showed that abundant ROS production could lead to increased peripheral Tregs, an important suppressor of anti-tumor immunity [38]. In line with these evidence, we also found that supernatants from Jurkat and H9 cells with higher mtDNA content increased the frequency of Tregs in
PBMCs. Previous data have shown that high concentration of TGF- $\beta 1$ and low concentration of TNF- $\alpha$ facilitate the differentiation and expansion of Tregs $[39,40]$. Therefore, it is possible that elevated mtDNA content induces ROS production and thus affect the secretion of cytokines, leading to increased Tregs in PBMCs. Furthermore, ROS can also induce NK cell dysfunction and apoptosis [41], and oxidative stress has been shown to induce the decrease of NK cell in PBMCs. In line with these findings, we confirmed that $\mathrm{H}_{2} \mathrm{O}_{2}$ or supernatants from Jurkat and H9 cells induced the decrease of NK cell frequency in PBMCs, probably via increased Tregs and TGF- $\beta 1$. Collectively, these data suggest that elevated mtDNA content in leukocytes might promote the progression of
A

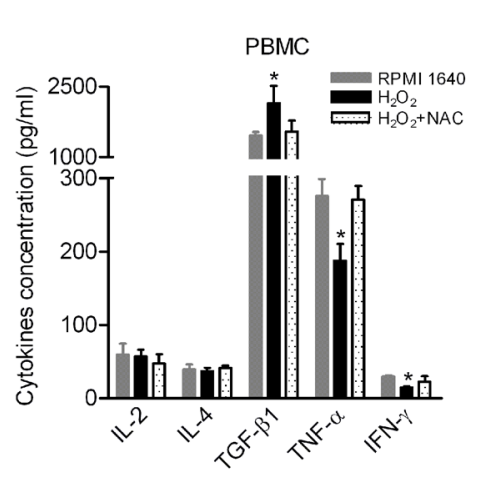

D

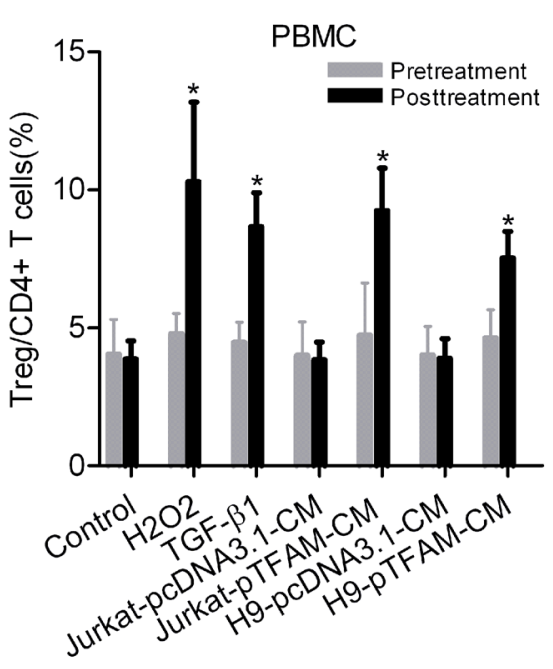

C

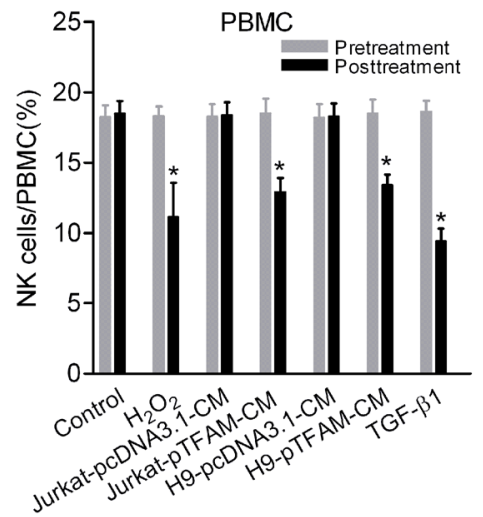

E

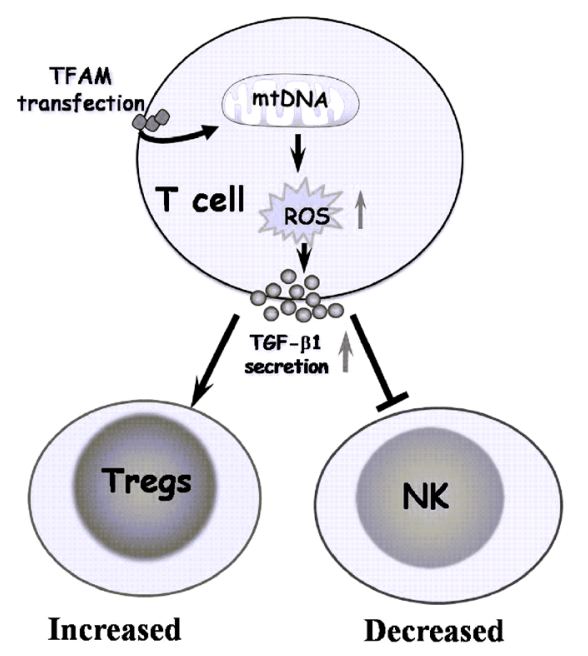

Figure 5: Elevated mtDNA content induced an immunosuppressive phenotype by ROS-mediated secretion of cytokines in PBMCs. A. Concentration of cytokines in culture supernatants measured by ELISA in PBMCs with treatment of $\mathrm{H}_{2} \mathrm{O}_{2}(100 \mu M)$ or both $\mathrm{H}_{2} \mathrm{O}_{2}(100 \mu \mathrm{M})$ and NAC $(20 \mathrm{mM})$ for 12 hours. B. Concentration of TNF- $\alpha$ and IFN- $\gamma$ measured by ELISA in PBMCs cultured with different conditional medium (CM). C. Frequency of NK cells from healthy donors measured by flow cytometry $48 \mathrm{~h}$ after different treatments. 50ng/ $\mathrm{mL}$ of TGF- $\beta 1$ was used when appropriate. D. Frequency of Treg cells in $\mathrm{CD} 4^{+} \mathrm{T}$ cells from healthy donors measured by flow cytometry in different treatments. E. A schematic diagram on the underlying mechanism of mtDNA content-mediated immunosuppression. ${ }^{*} P<0.05$, compared with related controls. 
HCC via ROS-induced immunosuppressive effects, which may explain the poor prognosis of these patients.

In summary, our findings indicate that leukocyte mtDNA content is an independent prognosis marker for HCC and can improve the prediction of TNM stage-based prognosis models. Mechanically, high mtDNA content may contribute to ROS-mediated immunosuppressive phenotype in the peripheral blood lymphocytes. Our study provides new insight into HCC pathological progression.

\section{MATERIALS AND METHODS}

\section{Patient population, sample and clinical data collection}

The eligibility criteria for patient recruitment were set as follows: (1) histologically-confirmed hepatocellular carcinoma (HCC); (2) receiving curative surgery; (3) only Child-Pugh A classification; (4) availability of complete clinical and follow-up data; (5) no preoperative anticancer treatment; (6) no history of other malignancy; and (7) alive at least 1 months after surgery. Finally, a total of 618 surgical HCC patients from two independent cohorts were included in the present study. Training cohort $(\mathrm{n}=113)$ was collected between March 2008 and September 2012 at Xijing Hospital affiliated with Fourth Military Medical University (FMMU) in Xi'an, China. Validation cohort (n $=505$ ) was collected between September 2009 and July 2013 at Eastern Hepatobiliary Surgery Hospital affiliated with Secondary Military Medical University (SMMU) in Shanghai, China. Before surgery, $5 \mathrm{ml}$ venous blood was collected from each patient and used for DNA extraction using the E.Z.N.A. blood DNA Midi Kit (Omega Bio-Tek, Norcross, GA). In addition, 40 additional HCC patients were enrolled between May 2014 and October 2014 from Tangdu Hospital of FMMU for immunoassays and $5 \mathrm{ml}$ venous blood was collected from each patient and separated by centrifugation. The plasma was used for cytokine assay and the blood cells were used for DNA extraction or isolation of peripheral blood mononuclear cells (PBMCs) by density gradient centrifugation over Ficoll-Hypaque (Amersham Pharmacia Biotech, NJ) as previously described [23]. PBMCs were also collected from 3 healthy donors from our lab. The demographic information, clinical and follow-up data of each patient was collected by well-trained staff interviewers or clinical specialists and described in Supplementary Methods. The latest follow-up date was January 2014 and the median follow-up duration was 33.4 months (ranging from 3.6 to 70 months). Overall survival was defined as the interval from surgery to death or last follow-up. Recurrence-free survival (RFS) was defined as the time from surgery to the date of the first recurrence or distant metastasis of HCC or death, whichever occurred first. The study was approved by the Ethic Committee of the Fourth Military Medical University and written informed consent was obtained from all participants.

\section{Detection of mtDNA content by real-time quantitative PCR}

Relative mtDNA content was measured by a twostep real-time quantitative PCR-based method. In the first step, the ratio of mtDNA copy number to HGB copy number was determined for each sample from standard curves. In the second step, the mtDNA/HGB ratio for each sample was normalized to a calibrator DNA to standardize between different runs and the normalized mtDNA/ $\mathrm{HGB}$ ratio was defined as the measurement of relative mtDNA content, which was affected by DNA sample used for standard curve, therefore only can be compared in this study. The detailed information was provided in Supplementary Methods.

\section{Cell culture}

T lymphoblast cell lines Jurkat and H9 were routinely maintained in RPMI 1640 medium supplemented with 10\% FBS (Thermo Scientific Hyclone). Cells were first activated by $1 \mu \mathrm{g} / \mathrm{ml}$ phytohemagglutinin (PHA) for $12 \mathrm{~h}$, washed, and subsequently cultured in RPMI 1640 medium for $24 \mathrm{~h}$. Then, the culture supernatant was filtered and collected for further immunoassays or used as conditioned medium (CM) for PBMC culturing. Fresh PBMCs were isolated from healthy donors. Conditioned culturing was performed in RPMI 1640 medium with $10 \%$ heat-inactivated FBS and 30\% culture supernatant from Jurkat or H9 cells. After $24 \mathrm{~h}$, cultured PBMCs and supernatant were collected and used for immunoassays.

\section{Plasmid construction and cell transfection}

The full-length DNA sequence encoding mitochondrial transcription factor A (TFAM) was cloned into pcDNA3.1(+) vector (defined as pTFAM) as previously described [24]. The recombinant or control plasmid was transfected into Jurkat or H9 cells using Lipofectamine 2000 reagent (Life Sciences) according to the manufacturer's instructions. The overexpression of TFAM was confirmed by Western blotting as described in Supplementary Methods.

\section{Immunophenotype analysis of PBMCs by flow cytometry}

Fresh or cultured PBMCs were fixed with 4\% formaldehyde and stained with fluorescence-conjugated antibodies against the following immune markers: CD3, CD4, CD8, CD25, FOXP3, CD19 and CD56 (BD Biosciences, NJ). Appropriate isotype controls were included for each sample. The immunophenotype detection was performed on a FACScan flow cytometer (Becton Dickinson, Franklin Lakes, NJ). 


\section{Concentration detection of cytokines by enzyme- linked immunoassay (ELISA)}

The concentrations of interleukin (IL)-2, IL-4, transforming growth factor (TGF)- $\beta 1$, tumor necrosis factor (TNF)- $\alpha$ and interferon (IFN)- $\gamma$ were detected in plasma of HCC patients or culturing supernatant by using ELISA kits (eBioscience, San Diego, CA) according to the manufacturer's instructions.

\section{Detection of ROS by flow cytometry}

Cellular reactive oxygen species (ROS) were detected by the fluorescent probe $\mathrm{H}_{2}$ DCFH-DA (Beyotime, shanghai, China) according to the manufacturer's protocols. Briefly, $\mathrm{H}_{2}$ DCFH-DA was diluted to a final concentration of $10 \mu \mathrm{M}$ with serum free medium. Then suspension of $\mathrm{H} 9$ and Jurkat cells transfected with different expression plasmids was incubated with DCFH$\mathrm{DA}$ at $37^{\circ} \mathrm{C}$ for 30 minutes and then analyzed on a FACScan flow cytometer (Becton Dickinson, Franklin Lakes, NJ) with an excitation wavelength of $488 \mathrm{~nm}$ and an emission wavelength of $535 \mathrm{~nm}$.

\section{Statistical analysis}

Chi-squared test was used to examine differences of categorical variables between subgroups. Student's $t$-test was used to analyze the difference of normally distributed continuous variables between two groups, while MannWhitney U test and Kruskal-Wallis H test were employed for the comparison of abnormally distributed continuous variables. The receiver operating characteristic curve analysis was used to select a leukocyte mtDNA content cutoff point for OS and RFS in the training cohort as previously described [25]. Kaplan-Meier survival curve was plotted and compared with a log-rank test. Multivariate Cox proportional hazards regression model was used to calculate the hazard ratio and $95 \%$ confidence interval for the association of clinicopathological variables and mtDNA content with OS and RFS of HCC patients. All statistical analyses were performed using the IBM SPSS Statistics 19.0 software (IBM), and $P<0.05$ was considered statistically significant.

\section{FUNDING}

This work was supported by National Natural Science Foundation of China (81171966, 81320108021) and International Science and Technology Cooperation Program of China (2013DFA32110).

\section{CONFLICTS OF INTEREST}

The authors have no conflicts of interest to disclose.

\section{REFERENCES}

1. Venook AP, Papandreou C, Furuse J, de Guevara LL. The incidence and epidemiology of hepatocellular carcinoma: a global and regional perspective. The oncologist. 2010; 15:5-13.

2. Dhanasekaran R, Limaye A, Cabrera R. Hepatocellular carcinoma: current trends in worldwide epidemiology, risk factors, diagnosis, and therapeutics. Hepatic medicine: evidence and research. 2012; 4:19-37.

3. Suriawinata A, Xu R. An update on the molecular genetics of hepatocellular carcinoma. Seminars in liver disease. 2004; 24:77-88.

4. Wallace DC, Fan W, Procaccio V. Mitochondrial energetics and therapeutics. Annual review of pathology. 2010; $5: 297-348$.

5. Anderson S, Bankier AT, Barrell BG, de Bruijn $\mathrm{MH}$, Coulson AR, Drouin J, Eperon IC, Nierlich DP, Roe BA, Sanger F, Schreier PH, Smith AJ, Staden R, Young IG. Sequence and organization of the human mitochondrial genome. Nature. 1981; 290:457-465.

6. Chan DC. Mitochondria: dynamic organelles in disease, aging, and development. Cell. 2006; 125:1241-1252.

7. Lee HC, Wei YH. Mitochondrial DNA instability and metabolic shift in human cancers. International journal of molecular sciences. 2009; 10:674-701.

8. Lee HC, Li SH, Lin JC, Wu CC, Yeh DC, Wei YH. Somatic mutations in the D-loop and decrease in the copy number of mitochondrial DNA in human hepatocellular carcinoma. Mutation research. 2004; 547:71-78.

9. Wu CW, Yin PH, Hung WY, Li AF, Li SH, Chi CW, Wei YH, Lee HC. Mitochondrial DNA mutations and mitochondrial DNA depletion in gastric cancer. Genes, chromosomes \& cancer. 2005; 44:19-28.

10. Kim MM, Clinger JD, Masayesva BG, Ha PK, Zahurak ML, Westra WH, Califano JA. Mitochondrial DNA quantity increases with histopathologic grade in premalignant and malignant head and neck lesions. Clinical cancer research. 2004; 10:8512-8515.

11. Lin PC, Lin JK, Yang SH, Wang HS, Li AF, Chang SC. Expression of beta-F1-ATPase and mitochondrial transcription factor $\mathrm{A}$ and the change in mitochondrial DNA content in colorectal cancer: clinical data analysis and evidence from an in vitro study. International journal of colorectal disease. 2008; 23:1223-1232.

12. Yu M, Zhou Y, Shi Y, Ning L, Yang Y, Wei X, Zhang N, Hao X, Niu R. Reduced mitochondrial DNA copy number is correlated with tumor progression and prognosis in Chinese breast cancer patients. IUBMB life. 2007; 59:450-457.

13. Mizumachi T, Suzuki S, Naito A, Carcel-Trullols J, Evans TT, Spring PM, Oridate N, Furuta Y, Fukuda S, Higuchi M. Increased mitochondrial DNA induces acquired docetaxel resistance in head and neck cancer cells. Oncogene. 2008; $27: 831-838$. 
14. Hsu CW, Yin $\mathrm{PH}$, Lee $\mathrm{HC}$, Chi CW, Tseng LM. Mitochondrial DNA content as a potential marker to predict response to anthracycline in breast cancer patients. The breast journal. 2010; 16:264-270.

15. Yamada S, Nomoto S, Fujii T, Kaneko T, Takeda S, Inoue $\mathrm{S}$, Kanazumi N, Nakao A. Correlation between copy number of mitochondrial DNA and clinico-pathologic parameters of hepatocellular carcinoma. European journal of surgical oncology. 2006; 32:303-307.

16. Hashad DI, Elyamany AS, Salem PE. Mitochondrial DNA Copy Number in Egyptian Patients with Hepatitis C VirusRelated Hepatocellular Carcinoma. Genetic testing and molecular biomarkers. 2015; 19:604-609.

17. Zhao S, Yang Y, Liu J, Liu H, Ge N, Yang H, Zhang H, Xing J. Association of mitochondrial DNA content in peripheral blood leukocyte with hepatitis B virus-related hepatocellular carcinoma in a Chinese Han population. Cancer science. 2011; 102:1553-1558.

18. Xing J, Chen M, Wood CG, Lin J, Spitz MR, Ma J, Amos CI, Shields PG, Benowitz NL, Gu J, de Andrade M, Swan GE, Wu X. Mitochondrial DNA content: its genetic heritability and association with renal cell carcinoma. Journal of the National Cancer Institute. 2008; 100:1104-1112.

19. Hosgood HDIII, Liu CS, Rothman N, Weinstein SJ, Bonner MR, Shen M, Lim U, Virtamo J, Cheng WL, Albanes D, Lan Q. Mitochondrial DNA copy number and lung cancer risk in a prospective cohort study. Carcinogenesis. 2010; $31: 847-849$

20. Qu F, Liu X, Zhou F, Yang H, Bao G, He X, Xing J. Association between mitochondrial DNA content in leukocytes and colorectal cancer risk: a case-control analysis. Cancer. 2011; 117:3148-3155.

21. Xia P, An HX, Dang CX, Radpour R, Kohler C, Fokas E, Engenhart-Cabillic R, Holzgreve W, Zhong XY. Decreased mitochondrial DNA content in blood samples of patients with stage I breast cancer. BMC cancer. 2009; 9:454.

22. Qu F, Chen Y, Wang X, He X, Ren T, Huang Q, Zhang J, Liu X, Guo X, Gu J, Xing J. Leukocyte mitochondrial DNA content: a novel biomarker associated with prognosis and therapeutic outcome in colorectal cancer. Carcinogenesis. 2015; 36:543-552.

23. Yokokawa J, Cereda V, Remondo C, Gulley JL, Arlen PM, Schlom J, Tsang KY. Enhanced functionality of CD4+CD25(high)FoxP3+ regulatory $\mathrm{T}$ cells in the peripheral blood of patients with prostate cancer. Clinical cancer research. 2008; 14:1032-1040.

24. Suarez J, Hu Y, Makino A, Fricovsky E, Wang H, Dillmann WH. Alterations in mitochondrial function and cytosolic calcium induced by hyperglycemia are restored by mitochondrial transcription factor A in cardiomyocytes. American journal of physiology Cell physiology. 2008; 295:C1561-1568.
25. Zlobec I, Vuong T, Hayashi S, Haegert D, Tornillo L, Terracciano L, Lugli A, Jass J. A simple and reproducible scoring system for EGFR in colorectal cancer: application to prognosis and prediction of response to preoperative brachytherapy. British journal of cancer. 2007; 96:793-800.

26. Nadkarni S, Mauri C, Ehrenstein MR. Anti-TNF-alpha therapy induces a distinct regulatory $\mathrm{T}$ cell population in patients with rheumatoid arthritis via TGF-beta. The Journal of experimental medicine. 2007; 204:33-39.

27. Thyagarajan B, Wang R, Nelson H, Barcelo H, Koh WP, Yuan JM. Mitochondrial DNA copy number is associated with breast cancer risk. PloS one. 2013; 8:e65968.

28. Lynch SM, Weinstein SJ, Virtamo J, Lan Q, Liu CS, Cheng WL, Rothman N, Albanes D, Stolzenberg-Solomon RZ. Mitochondrial DNA copy number and pancreatic cancer in the alpha-tocopherol beta-carotene cancer prevention study. Cancer prevention research. 2011; 4:1912-1919.

29. Cheng AL, Kang YK, Chen Z, Tsao CJ, Qin S, Kim JS, Luo R, Feng J, Ye S, Yang TS, Xu J, Sun Y, Liang H, et al. Efficacy and safety of sorafenib in patients in the AsiaPacific region with advanced hepatocellular carcinoma: a phase III randomised, double-blind, placebo-controlled trial. The Lancet Oncology. 2009; 10:25-34.

30. Korangy F, Hochst B, Manns MP, Greten TF. Immune responses in hepatocellular carcinoma. Digestive diseases. $2010 ; 28: 150-154$.

31. Lee IC, Huang YH, Chau GY, Huo TI, Su CW, Wu JC, Lin HC. Serum interferon gamma level predicts recurrence in hepatocellular carcinoma patients after curative treatments. International journal of cancer. 2013; 133:2895-2902.

32. Chew V, Tow C, Teo M, Wong HL, Chan J, Gehring A, Loh M, Bolze A, Quek R, Lee VK, Lee KH, Abastado JP, Toh HC, Nardin A. Inflammatory tumour microenvironment is associated with superior survival in hepatocellular carcinoma patients. Journal of hepatology. 2010; 52:370-379.

33. Giannelli G, Villa E, Lahn M. Transforming growth factorbeta as a therapeutic target in hepatocellular carcinoma. Cancer research. 2014; 74:1890-1894.

34. Murphy MP. How mitochondria produce reactive oxygen species. The Biochemical journal. 2009; 417:1-13.

35. Lee $\mathrm{HC}$, Yin $\mathrm{PH}, \mathrm{Lu} \mathrm{CY}$, Chi CW, Wei YH. Increase of mitochondria and mitochondrial DNA in response to oxidative stress in human cells. The Biochemical journal. 2000; 348 Pt 2:425-432.

36. Jain M, Rivera S, Monclus EA, Synenki L, Zirk A, Eisenbart J, Feghali-Bostwick C, Mutlu GM, Budinger GR, Chandel NS. Mitochondrial reactive oxygen species regulate transforming growth factor-beta signaling. The Journal of biological chemistry. 2013; 288:770-777.

37. Yang Y, Bazhin AV, Werner J, Karakhanova S. Reactive oxygen species in the immune system. International reviews of immunology. 2013; 32:249-270. 
38. Kraaij MD, Savage ND, van der Kooij SW, Koekkoek K, Wang J, van den Berg JM, Ottenhoff TH, Kuijpers TW, Holmdahl R, van Kooten C, Gelderman KA. Induction of regulatory $\mathrm{T}$ cells by macrophages is dependent on production of reactive oxygen species. Proceedings of the National Academy of Sciences of the United States of America. 2010; 107:17686-17691.

39. Zhang Q, Cui F, Fang L, Hong J, Zheng B, Zhang JZ. TNFalpha impairs differentiation and function of TGF-betainduced Treg cells in autoimmune diseases through Akt and Smad3 signaling pathway. Journal of molecular cell biology. 2013; 5:85-98.
40. Nie H, Zheng Y, Li R, Guo TB, He D, Fang L, Liu X, Xiao L, Chen X, Wan B, Chin YE, Zhang JZ. Phosphorylation of FOXP3 controls regulatory $\mathrm{T}$ cell function and is inhibited by TNF-alpha in rheumatoid arthritis. Nature medicine. 2013; 19:322-328.

41. Mellqvist UH, Hansson M, Brune M, Dahlgren C, Hermodsson S, Hellstrand K. Natural killer cell dysfunction and apoptosis induced by chronic myelogenous leukemia cells: role of reactive oxygen species and regulation by histamine. Blood. 2000; 96:1961-1968. 